\title{
Mémoire espagnole et mémoire indienne dans un présage aztèque
}

Nathalie Ragot

\section{OpenEdition}

12 Journals

Édition électronique

URL : https://journals.openedition.org/jsa/3703

DOI : 10.4000/jsa.3703

ISSN : 1957-7842

Éditeur

Société des américanistes

Édition imprimée

Date de publication : 1 janvier 2003

Pagination : 9-20

ISSN : 0037-9174

\section{Référence électronique}

Nathalie Ragot, "Mémoire espagnole et mémoire indienne dans un présage aztèque ", Journal de la Société des américanistes [En ligne], 89-1 | 2003, mis en ligne le 16 janvier 2008, consulté le 02 septembre 2022. URL : http://journals.openedition.org/jsa/3703 ; DOI : https://doi.org/10.4000/jsa. 3703 


\title{
ARTICLES
}

\section{MÉMOIRE ESPAGNOLE ET MÉMOIRE INDIENNE DANS UN PRÉSAGE AZTÈQUE ${ }^{1}$}

\author{
Nathalie RAGOT *
}

Cet article se propose d'analyser un exemple des problèmes qui peuvent survenir dans le processus de formation de la mémoire écrite. À travers l'étude comparative de deux textes relatant le même événement, un présage aztèque annonciateur de la Conquête, nous suivrons l'évolution des éléments constitutifs du récit et leur manipulation consciente et/ou inconsciente. [MOTS CLÉs : ethno-histoire, présage, aztèque, mémoire, Conquête.]

Spanish and Indian Memories in an Aztec Omen. The present article analyses an example of the problems which could occur in the process of the written memory construction. Through the comparative study of two versions of the same event, an Aztec omen about the Conquest, we'll follow the evolution of the constituent elements of the account and their conscious and/or unconscious manipulation. [KEY wORDS : ethno-history, omen, Aztec, memory, Conquest.]

Memoria española y memoria indigena en un presagio azteca. Este artículo ofrece un análisis de un ejemplo de los problemas que pueden surgir en el proceso de formación de la memoria escrita. A través del estudio comparativo de dos textos que relatan el mismo acontecimiento, es decir un presagio azteca anunciando la Conquista, seguiremos la evolución de los elementos constitutivos del relato y sus manipulaciones conscientes y/o inconscientes. [PALABRAS ClAVES : etno-historia, presagio, azteca, memoria, Conquista.]

Une prophétie aztèque ${ }^{2}$, annonciatrice de la Conquête, raconte l'étrange voyage dans l'au-delà d'une femme morte. Revenue bien vivante sur terre, elle alla

* Docteur en anthropologie religieuse, École pratique des hautes études, Paris [nathalie.ragot @wanadoo.fr].

Journal de la Société des Américanistes, 2003, 89-1 : p. 9 à 20. Copyright C Société des Américanistes. 
annoncer à Montezuma II, le tlatoani ${ }^{3}$ de Mexico-Tenochtitlan, la fin prochaine de son empire sous les coups d'envahisseurs inconnus. Le récit de cet extraordinaire événement a été consigné par deux chroniqueurs espagnols : Bernardino de Sahagún et Juan de Torquemada. Cependant, les deux versions, que seules quelques décennies séparent, présentent de notables différences qui nous ont conduits à nous interroger, non seulement sur la façon dont se manifestent ces dissemblances mais aussi sur leurs raisons, en cherchant à reconstituer l'histoire de la formation de ces deux récits. Quelles étaient les motivations des rédacteurs et des informateurs? Les différences entre les deux versions résultent-elles d'une action volontaire ou inconsciente du récitant ou du consignant ? etc. Pour répondre à ces interrogations, qui relèvent plus du vaste problème de la collecte, de l'enregistrement, de la conception, de l'évolution et/ou de la manipulation de la mémoire au travers des traces écrites, nous procéderons en deux étapes. Tout d'abord, par l'analyse comparative des deux récits, nous soulignerons l'évolution de leurs éléments symboliques et les processus d'interpolation et de métissage qui s'y sont glissés. Ensuite, l'évaluation de l'histoire de la ressuscitée dans son contexte historique nous permettra de montrer que, à l'instar d'autres présages, elle atteste une tentative indigène d'intégrer l'événement de la Conquête dans un schéma de lecture connu et correspondant à une conception cyclique de l'histoire dont le modèle a déjà été exploité dans le passé mythique de la tribu.

\section{PRÉSENTATION DES DEUX VERSIONS DE LA PROPHÉTIE}

Le récit le plus ancien apparaît dans les folios $84 \mathrm{r}^{\circ}-\mathrm{v}^{\circ}$ du manuscrit des Primeros Memoriales consignés par le frère Bernardino de Sahagún durant son séjour à Tepepulco entre 1559 et 1561 . Sahagún présente ce paragraphe comme une « histoire miraculeuse ou prophétie survenue à Mexico pendant le règne de Montezuma, dernier du nom, dix ou douze ans avant l'arrivée des Espagnols dans ce pays » (Primeros Memoriales 1997, pp. 179-183). Rédigé en nahuatl, le texte relate comment Quetzalpetlatl, la sœur de Montezuma II, morte de diarrhée, est cérémonieusement enterrée. Après quatre jours et quatre nuits passés dans sa sépulture, un jeune homme apparaît à la défunte pour la guider jusque dans l'au-delà dénommé Tlalocan. Rendu en ce lieu, le dieu Tlaloc lui montre son monde baignant dans la brume et ses différents habitants. Il lui donne une petite boîte et une calebasse en lui indiquant que c'est ce qu'elle doit manger et boire et qu'avec cela elle pourra aider les gens sur terre (ibid., pp. 179-183; 1907, fol. 111112 ; Anderson 1988, pp. 155-159). Le récit s'interrompt brutalement faute du folio suivant malheureusement perdu, mais une version extrêmement résumée est rapportée dans le Codex de Florence, nous éclairant ainsi sur la fin de l'histoire (Codex de Florence 1950-1981, VIII, p. 3 ; Anderson 1988, pp. 159-160). Ce court passage rapporte qu'une femme noble tenochca (son nom n'est pas précisé) 
mourut puis revint dans le monde des vivants pour prédire à Montezuma la fin prochaine de son empire (Codex de Florence 1950-1981, VIII, p. 3).

La deuxième relation de cette prophétie, plus tardive, apparaît dans l'ouvrage du frère Juan de Torquemada, Monarquía Indiana, imprimé à Séville en 1615. Torquemada a recueilli des données au Mexique durant le dernier quart du Xvi ${ }^{\mathrm{e}}$ siècle mais il a surtout fait œuvre de compilateur en puisant abondamment dans les travaux de ses prédécesseurs. En ce qui concerne l'histoire de la ressuscitée, tout laisse à penser qu'il s'est directement inspiré des écrits de Sahagún mais en y apportant sa «touche personnelle » (Torquemada 1975-1983, vol. VII). Dans cette version, la défunte, appelée Papán (ou Papantzin), est enterrée puis revient sur terre pour raconter à son frère Montezuma son voyage dans l'au-delà. On peut la résumer ainsi : de sa sépulture elle se réveilla dans une grande plaine coupée en son milieu par un large fleuve. Quand elle voulut le traverser; elle vit apparaître un jeune homme qui lui proposa de la guider. Le suivant, elle vit des ossements entassés qui étaient en train de crier et des hommes noirs avec des cornes en train de construire une maison. En regardant vers l'est, elle vit un grand bateau avec des individus à la peau rose vêtus d'étrange manière et se présentant comme les fils du Soleil. Son compagnon lui expliqua que ces hommes amenaient une nouvelle foi et qu'ils seraient les nouveaux maîtres du pays. Il lui dit aussi qu'elle devait être la représentante de cette nouvelle foi sur la terre et guider ses compatriotes vers celle-ci (Torquemada 1986, t. I, pp. 237-239).

L'histoire est bien la même, mais les composantes du récit ont changé. Voyons comment ces évolutions se manifestent et quelles en sont les conséquences quant à l'impact du récit sur son " public ».

\section{ANALYSE COMPARATIVE DES ÉLÉMENTS CONSTITUTIFS DU RÉCIT}

\section{Décès et rituel funéraire}

D’après les Primeros Memoriales, Quetzalpetlatl meurt de diarrhée. Pour les Aztèques, cette façon de mourir, de même que la noyade, le foudroiement, la lèpre, la goutte, l'hydropisie, etc., était considérée comme caractéristique de Tlaloc, divinité liée à la fertilité et aux précipitations (Sahagún 1992, p. 207, 357 ; Codex de Florence 1950-1981, III, p. 47 ; VI, p. 115 ; Mendieta 1980, pp. 96-97 ; Torquemada 1986, II, p. 82, 529). Dans les croyances aztèques, la façon de mourir était généralement révélatrice du destin du défunt dans l'autre monde. Ainsi, ceux qui mouraient d'une mort associée à Tlaloc allaient le rejoindre dans son au-delà, le Tlalocan, où ils devenaient des Tlaloques à son service. Leurs dépouilles, contrairement à celles d'autres défunts, n'étaient pas brûlées mais enterrées (Sahagún 1992, p. 207 ; Codex de Florence 1950-1981, III, p. 47). Cette différence de traitement funéraire en fonction du mode de trépas est explicite dans le premier 
texte du présage : " [...] Quand elle mourut les anciens dirent : "Notre nièce est morte. Brûlons-la". Mais alors certains dirent : "Elle ne doit pas être incinérée, nous devons l'enterrer". Et la décision qu'ils devaient l'enterrer fut unanime » (Primeros Memoriales 1997, p. 180). Suit une description assez détaillée de la façon dont le corps est mis en position fléchie, enroulé dans de nombreuses pièces de tissus et paré de multiples ornements de papier pour être déposé dans une fosse profonde. Ces importants éléments sur le mode de traitement et la préparation du cadavre ont disparu de la version de Torquemada dans laquelle Papán meurt d'une anonyme et "grave maladie », qui n'implique plus ni au-delà ni rituel funéraire particulier.

Dans la version des Primeros Memoriales, Quetzalpetlatl reste quatre jours et quatre nuits dans sa tombe avant d'entreprendre son voyage dans l'au-delà. Ce laps de temps est significatif puisque, dans les croyances anciennes, le temps nécessaire au mort pour quitter la terre et s'en aller dans l'autre monde était justement de quatre jours (López Austin 1984, I, pp. 364-365 ; Ragot 1999, p. 191). Dans le récit de Torquemada, ledit temps a changé, perdant ainsi toute signification symbolique ; il n'est plus que d'une après-midi et d'une seule nuit.

\section{L'apparition}

Le jeune homme qui guide la défunte dans l'au-delà subit des modifications significatives d'un texte à l'autre. Dans la version de Sahagún, Quetzalpetlatl vit apparaître devant elle un jeune homme « maladif et déformé » (Primeros Memoriales 1997, p. 180). C'est un envoyé de Tlaloc et de telles caractéristiques physiques correspondent bien à celles des défunts qui allaient au Tlalocan, c'est-à-dire les goutteux, les hydropiques, les lépreux, etc. (Codex de Florence 1950-1981, III, p. 47). Dans la version transmise par Torquemada, il a un tout autre aspect :

[...] Un jeune homme vêtu d'une longue tunique nette comme un cristal et rayonnante comme le soleil, son visage brillait comme une étoile ; sur son front se voyait une figure qu'il répétait en mettant un doigt sur un autre en forme de croix. Il avait des ailes en plumes riches nuancées de charmants reflets ; ses yeux brillaient comme des émeraudes et le regard était modeste. Ce personnage était blond, de bel aspect et de taille imposante. ${ }^{4}$ (Torquemada 1986, I, p. 238)

Le guide de Papán arbore toutes les caractéristiques d'une apparition angélique ! D'envoyé du dieu préhispanique Tlaloc, il est devenu un ange envoyé du dieu chrétien. D'ailleurs, Tlaloc, décrit comme vieux et couvert de caoutchouc liquide - détails iconographiques qui correspondent bien aux descriptions et aux représentations connues de cette divinité (Sahagún 1992, p. 886; Primeros Memoriales 1997, p. 97) -, a complètement disparu du récit de Torquemada où l'ange est l'unique interlocuteur de Papán. 


\section{Le voyage dans l'au-delà}

Avant d'arriver au Tlalocan, Quetzalpetlatl et son guide empruntent un chemin bien particulier :

[...] Il la guida dans le Pays des morts ; il lui fit suivre de grandes plaines, des prés inhabités. Quand il l'emmena là, ils virent alors des lézards ; ils allèrent là où il y a plein de pierres de chaque côté [et] ils traversèrent. Et aussi, plus loin, ils traversèrent où les femmes étaient en train de tisser. Et alors il l'emmena au Tlalocan. Là ils passèrent où étaient les grenouilles, comme au printemps. Elles étaient assises sur un mur ; il y avait là deux grenouilles. À partir de là étaient étendues des bannières tachetées de caoutchouc liquide, avec lesquelles s'achevait le chemin. (Primeros Memoriales 1997, pp. 180-181)

Cette précieuse description rappelle immédiatement certaines étapes du voyage que tous les défunts, selon les croyances pré-hispaniques, devaient accomplir dans l'au-delà. Entre autres épreuves, ils devaient emprunter un chemin gardé par un lézard bleu, appelé xochitonal, errer dans des lieux inhospitaliers, le Chicuey ixtlaoatl, " huit déserts ", et le Chicuetiliuhcan, " huit montagnes ", ou encore passer par le Pancuecuetlacayan, « lieu où flottent les drapeaux « (Codex de Florence 1950-1981, III, pp. 43-44 ; Sahagún 1992, pp. 206-207 ; Codex Vaticamus A 1965, pl. 2, pp. 10-11 ; Ragot 1999, pp. 208-211). Le chemin emprunté par Quetzalpetlatl est conçu sur le même modèle que celui du voyage du défunt dans l'inframonde préhispanique, ce qui ne doit pas nous surprendre puisqu'elle est censée être morte et en route vers sa dernière demeure. Dans la version de Torquemada, ce voyage n'existe plus. De sa tombe Papán reprend conscience :

[...] Dans une spacieuse vallée qui paraissait n'avoir ni commencement ni fin, très plate, dépourvue de coteaux, de ravins et de montagnes. Vers son milieu se trouvait un chemin qui bientôt se divisait en sentiers divers. Sur l'un des côtés de la vallée coulait une rivière considérable. (Torquemada 1986, t. I, p. 238)

À l'inverse de ce qui est décrit dans la version des Primeros Memoriales, les accidents de relief sont, dans celle de Torquemada, gommés du paysage et l'accent est mis sur la platitude du lieu. Seul le large fleuve pourrait renvoyer à l'étape du passage du fleuve des croyances anciennes (Codex de Florence 19501981, III, pp. 43-44 ; Sahagún 1992, pp. 206-207 ; Codex Vaticanus A 1965, pl. 2, pp. 10-11), mais il pourrait tout aussi bien correspondre au Styx, le plus grand des fleuves des Enfers.

\section{Description de l'au-delà}

De la version de Torquemada ont également disparu les descriptions de l'au-delà Tlalocan et les multiples détails concernant ses habitants, ce qui repré- 
sentait pourtant plusieurs paragraphes dans la version des Primeros Memoriales. À leur place, Torquemada propose clairement une esquisse de l'enfer, du moins d'un sombre purgatoire, où seront damnés les païens d'hier et de demain, ceux déjà morts et ceux qui tomberont dans les futurs affrontements avec les Espagnols. Comme l'explique clairement son guide à Papán, en lui commentant ce qu'elle voit autour d'elle :

[...] Ces ossements et ces têtes que l'on entendait gémir étaient leurs ancêtres qui n'avaient pas eu les lumières de la foi, ce qui était cause de leur tourment ; que cette maison élevée par les Noirs était destinée à enfermer ceux qui mourraient dans les batailles qu'on allait soutenir contre les gens des navires. (Torquemada 1986, t. I, p. 239)

On le voit, la version rédigée par Torquemada est complètement revisitée suivant un environnement symbolique et une eschatologie chrétienne. Autre manifestation de cette réécriture : les propos des Indiens non encore évangélisés contiennent déjà des références à dieu et au diable. Par exemple, en voyant sa sœur revenue du monde des morts, Montezuma s'écrie : « Est-ce bien toi ma sœur ou n'est-ce que le démon déguisé dans ta personne? » (ibid., p. 238).

\section{La prophétie}

Dans le récit de Torquemada, il est clair que Papán a été envoyée dans l'au-delà afin d'annoncer à Montezuma la venue des Espagnols. Elle revient sur terre pour lui faire le récit de sa vision de grands navires qui viennent de l'Orient en transportant des hommes à la peau claire, se présentant comme les fils du Soleil destinés à dominer cette terre après de grandes guerres. Mais, outre ce rôle d'annonciatrice, Papán est également investie d'une autre mission, qui consiste à donner l'exemple et mener ses compatriotes sur le chemin de la nouvelle foi professée par les envahisseurs :

[...] Le jeune [...] me dit [...] que j'étais réservée à voir de mes propres yeux les événements prendre une autre tournure et à jouir des avantages de la foi dont ces personnages étaient porteurs ; que je les attendisse donc pour être témoin des guerres qui allaient s'engager entre eux et nous et les voir devenir maîtres de ces royaumes. [...] Que je revinsse sur mes pas pour les attendre, afin que, lorsque le calme s'établirait et que les ablutions du baptême seraient annoncées, je fusse la première à guider mes compatriotes qui devaient en profiter. (ibid., p. 239)

Le texte ajoute qu'elle fut la première habitante de Tlatelolco à être baptisée. Dans la version incomplète des Primeros Memoriales les choses ne sont pas si explicites. Si l'on en croit la version résumée qui apparaît dans le Codex de Florence, c'est également pour divulguer la nouvelle de l'arrivée d'envahisseurs que Quetzalpetlatl est envoyée au Tlalocan : 
[...] Et cette femme, après être revenue à la vie, alla parler avec Montezuma au sujet de ce qu'elle avait vu. Elle l'informa et lui dit : « Pour cette raison je suis revenue à la vie : je suis venue te dire que ton artifice tire à sa fin. Avec toi le règne cessera ; en ton temps se terminera la ville de Mexico. Ceux qui arrivent, ceux-là soumettront le pays ; ils occuperont Mexico ». (Codex de Florence 1950-1981, VIII, p. 3)

Il est très possible que la partie manquante de la version des Primeros Memoriales relatât également une vision des conquérants et l'annonce de leur future victoire sur les autochtones, ce qui semble être le but de la prophétie. Si l'on ne peut être affirmatif sur ce point, en revanche, il semblerait qu'un rôle d'émissaire de la nouvelle foi, similaire à celui dévolu à Papán, ait été également attribué à Quetzalpetlatl. En effet, selon le texte des Primeros Memoriales, Tlaloc dépose une petite boîte sur sa poitrine et lui donne une calebasse bleu-vert sans préciser leur contenu mais en lui expliquant clairement leur fonction :

[...] Ceci est ce que tu dois manger, ce que tu dois boire. Avec cela tu consoleras les gens sur la terre. Nulle part tu n'offenseras le seigneur du Tlalocan. Ceci n'est pas seulement pour les gens prospères qui vivent dans l'abondance, avec ceci tu aideras tous ceux qui sont dans la misère. Ainsi il lui ordonna. (Primeros Memoriales 1997, pp. 182-183, 1907, fol. 111-112; Anderson 1988, p. 159)

Nous pensons que les objets que Tlaloc remet à Quetzalpetlatl peuvent être identifiés aux emblèmes de la communion. Il n'est certes pas dit explicitement qu'il s'agisse du corps et du sang du Christ ; ils sont ce que la défunte doit boire et manger et ce avec quoi elle pourra aider les malheureux ! Ainsi, comme dans la version de Torquemada, Quetzalpetlatl sera un instrument divin, en offrant à ses compatriotes les symboles de la communion et la foi nouvelle qu'ils représentent.

Si cette interprétation est exacte, comment et pourquoi un tel message, explicite et sans surprise dans le texte de Torquemada, apparaît-il dans la version des Primeros Memoriales qui, pour le reste, semble avoir peu subi une acculturation? Notons également que c'est Tlaloc, un des dieux précolombiens les plus anciens et sûrement les plus honorés dans la région de l'Altiplano, qui prononce ces paroles et investit Quetzalpetlatl de sa mission. Doit-on voir dans ce passage une intervention volontaire de la part du chroniqueur pour aider à la conversion? Est-on face à un phénomène de modification partielle de textes originaux, comparable à ce qui s'est parfois produit avec des Huehuetlahtolli ou des chants poétiques dans lesquels les noms des divinités locales ont pu être remplacés par ceux de Dieu ou de la Vierge Marie? (León-Portilla y Silva Galena 1991, pp. 37-38 ; Garibay 1964-1966 ; Bierhorst 1985). À l'appui de l'hypothèse d'une manipulation du texte à des fins d'évangélisation, on doit se rappeler qu'historiquement le processus de conversion est passé d'abord par l'élite, laquelle donnait l'exemple, et c'est justement ce rôle qui est assigné aussi bien à Quetzalpetlatl qu'à 
Papán. Et, dans les deux cas, la défunte est la sœur de Montezuma, lien de parenté qui la place au sommet de la pyramide sociale.

Au vu de tout l'environnement préhispanique dans lequel s'ancre le récit des Primeros Memoriales, la précision et la justesse des détails, nous pouvons supposer qu'il a été rédigé, du moins récité, par un Indien, bon connaisseur de son héritage précolombien. Dans cette optique, la présence des symboles de la communion dans le texte pourrait être un acte volontaire dont les motivations devraient s'éclairer grâce à la genèse même de cette histoire.

\section{GENÈSE DU RÉCIT DES PRIMEROS MEMORIALES}

Nous avons mentionné dans l'introduction que le récit de la ressuscitée Quetzalpetlatl-Papán appartient à tout un groupe de signes annonciateurs de la Conquête. Ce type de témoignage n'est pas exclusif des Aztèques, il en existe aussi chez les Mayas, les Tlaxcaltèques, les Tarasques ou encore dans les sources relatives au Pérou (Muñoz Camargo 1892, pp. 167-173 ; Relation de Michoacan 1984, pp. 257-265; Todorov 1982, pp. 79-80). Relatant des faits censés s'être produits avant la Conquête, ces présages se sont pourtant diffusés après l'événement et sont le résultat de diverses influences. Comme l'a justement noté Michel Graulich :

[...] En gros, on peut discerner deux groupes d'intersignes : ceux d'influence espagnole, édifiants ou destinés à justifier la Conquête; et ceux d'origine aztèque, simplement annonciateurs, ou cherchant à expliquer la défaite, mais tendant, dans l'un et l'autre cas, à récuser le totalement nouveau, à le présenter comme prévu et attendu dans la conception cyclique de l'histoire. (Graulich 1991, p. 139 ; voir aussi Todorov 1982, pp. 72-99 ; Baudot et Todorov 1983, pp. 366-372)

Dans les présages de la première catégorie apparaissent des éléments totalement espagnols (par exemple la description de caractéristiques physiques des conquistadores, l'intervention de Dieu ou du démon, la manifestation de notions comme la conversion et le salut des âmes, la fin des sacrifices humains, etc.). À l'inverse, les présages de la seconde catégorie se composent de récits où apparaissent des signes traditionnels, par exemple les comètes, les colonnes de feu, les guerriers se battant dans le ciel... (Graulich 1991, pp. 139-143).

C'est cette volonté d'enraciner dans le passé un événement nouveau, de le lire au travers d'un schéma connu, afin de pouvoir l'intégrer ou l'accepter, qui peut nous donner une clé pour comprendre la présence des symboles de la communion dans la version des Primeros Memoriales. En fait, il s'avère que cette prophétie est construite suivant un modèle déjà utilisé dans le mythe fondateur mexica : les récits de la pérégrination et, plus précisément, la partie concernant la fin de l'errance et l'arrivée à la « terre promise », là où se construira Tenochtitlan. Selon 
le mythe, les Mexicas, suivant les ordres de leur dieu tutélaire Huitzilopochtli, abandonnèrent leur île originelle Aztlan pour partir en quête d'une nouvelle terre où s'installer. Le Codex Aubin, récit de cette quête, raconte que, quand les voyageurs arrivèrent au lieu promis, un prêtre nommé Axollohua se noya dans l'eau de la lagune. Le jour suivant, il revint vivant auprès de ses compagnons et leur dit : «[...] Je suis allé voir Tlaloc, il m'a appelé et il m'a dit : mon fils Huitzilopochtli est arrivé, ceci est sa maison, il est le seul que l'on doit aimer et il restera avec moi dans ce monde » (Codex Aubin 1979, pp. 94-95). Le voyage d'Axollohua au Tlalocan doit être interprété comme une tentative de légitimation du pouvoir des nouveaux arrivants, les Mexicas (Graulich 1987, p. 242), et une intronisation de leur dieu tutélaire, Huitzilopochtli, par le dieu local le plus ancien, à savoir Tlaloc. À l'appui de cette interprétation, rappelons les tentatives avérées des nouveaux arrivants pour se forger une place dans l'histoire, que ce soit, comme l'ordonna le tlatoani Itzcoatl, en brûlant les anciens manuscrits figuratifs contenant les vieilles traditions afin de réécrire l'histoire en faveur des Mexicas, ou encore par les tentatives de substitution, dans les fêtes comme dans les mythes, de Huitzilopochtli à Quetzalcoatl (Graulich 1987, pp. 206-209, 347349 ; Codex de Florence 1950-1981, X, p. 191). Selon notre analyse, le récit de Quetzalpetlatl-Papán s'inscrit dans cette logique de remplacement de l'ancien occupant par le nouvel arrivant. La prophétie énoncée par la défunte, revenue elle aussi de chez Tlaloc qui était, répétons-le, le dieu le plus ancien et sûrement le plus révéré dans l'Altiplano, est comparable à celle d'Axollohua. Mais ici ce sont les Espagnols, derniers arrivés et vainqueurs, qui sont présentés comme les nouveaux seigneurs du pays et leur foi nouvelle comme celle à laquelle il faut désormais adhérer ${ }^{5}$. Le nouveau dieu des vainqueurs remplacera Huitzilopochtli, comme ce dernier avait succédé à Quetzalcoatl.

Un autre écho de la dimension cyclique de cette prophétie transparaît dans les noms mêmes des protagonistes, puisque Quetzalpetlatl et Papantzin apparaisent dans un autre mythe où c'est encore la chute d'un peuple, les Toltèques, qui est mise en scène. Dans les Anales de Cuauhtitlan, Quetzalcoatl est persécuté par Tezcatlipoca et tombe dans son piège en cessant ses devoirs de piété et en buvant le pulque qu'il lui offre. Saoul, Quetzalcoatl appelle sa sœur et passe la nuit avec elle. Le jour suivant, désespéré de ce qu'il a fait, il abandonne sa cité et s'en va en direction de l'est, vers sa mort (Anales de Cuauhtitlan 1945, pp. 8-11). Dans cette version du mythe, le nom de la sœur est Quetzalpetlatl. Comme elle, la ressuscitée des Primeros Memoriales est présentée comme la sœur de celui qui va déchoir, en l'occurrence Montezuma. Dans une autre version du mythe de la fin de Quetzalcoatl, l'homme qui fabrique le pulque, instrument fatal de la chute, se nomme Papantzin (Alva Ixtlilxochitl 1985, I, pp. 274-275), même nom que celui porté par la ressuscitée dans la version de Torquemada. Il est notable, et certainement pas accidentel, de rencontrer les mêmes noms de personnages dans des récits qui annoncent la chute d'un peuple, que ce soit les Toltèques avec Quetzalcoatl ou les 
Aztèques avec Montezuma. Malheureusement, on peut difficilement pousser la comparaison plus avant et force est d'avouer notre ignorance quant au pourquoi du choix de ces noms en relation avec de telles actions.

\section{CONCLUSION}

La comparaison des récits mettant en scène la ressuscitée QuetzalpetlatlPapán illustre différents processus d'évolution et/ou de manipulation de l'histoire indienne dans des sources écrites, qu'ils soient le fait, conscient ou inconscient, des Espagnols ou des Indiens. Au travers les différences qu'affichent les deux versions peuvent se lire des volontés et des motivations sous-jacentes opposées qui sont à l'origine de leur rédaction. La version transmise par Torquemada se compose uniquement de références symboliques chrétiennes (apparition angélique, description de personnages diaboliques avec cornes et pieds de cerfs, référence à Dieu et au diable, etc.) au détriment de l'indigène qui a totalement disparu. Publiée presque un siècle après la chute de Mexico, cette version s'inscrit dans une volonté d'expliquer la Conquête comme une entreprise menée sous les auspices divins.

À l'inverse, la version des Primeros Memoriales, la plus ancienne, est, sans grande surprise, celle qui présente les éléments les plus préhispaniques (mode de trépas/rituel funéraire/au-delà, temps pour quitter la terre, voyage dans l'inframonde, divinité Tlaloc, description de l'environnement du Tlalocan, des défunts qui y habitent, etc.). Le texte s'inscrit sans équivoque dans un fond préhispanique et devait encore constituer à l'époque un récit signifiant pour un indigène, même acculturé. Conçu à partir d'un schéma qui avait déjà fait ses preuves dans les mythes de la pérégrination, ce récit avait pour but d'expliquer et/ou de justifier la fin de l'ère aztèque et la nouvelle hégémonie espagnole. *

* Manuscrit reçu en juin 2002, accepté pour publication en mars 2003.

\section{NOTES}

1. Ce texte est tiré d'une intervention orale faite en espagnol, au congrès de la FIEALC, tenu à Tel Aviv en avril 1999 (actes non publiés).

2. Par " Aztèques » nous entendons l'ensemble des peuples qui vivaient dans le bassin de Mexico à la veille de la Conquête et appartenaient à la Triple Alliance dominée par les Mexicas.

3. Tlatoani, " celui qui parle », correspond au roi, au chef de l'État.

4. Toutes les citations ont été traduites à partir du texte original par l'auteur.

5. Notons également que ce type d'attitude n'était pas caractéristique des seuls Aztèques puisque un récit tout à fait comparable apparaît dans la Relation de Michoacan, chronique du territoire tarasque. L'un des présages, survenus avant l'arrivée des Espagnols, raconte qu'un pêcheur en train de pêcher fut attrapé par un caïman qui l'emmena dans sa tanière. Là, le caïman dit au pêcheur que les 
nouveaux hommes qui allaient diriger le monde étaient arrivés et il le chargea d'annoncer la nouvelle au roi tarasque (Relation de Michoacan 1984, pp. 264-265).

\section{RÉFÉRENCES CITÉES}

Alva IxtLilxóchitl Fernando de

1985 Obras Históricas, Edmundo O'Gorman, ed., 2 vols, IIH, UNAM, México, coll. « Historiadores y cronistas de Indias » 4.

Anales de Cuauhtitlan

1945 in Códice Chimalpopoca, Primo F. Velázquez, ed., Instituto de Investigaciones Antropológicas, UNAM, México.

ANDERSON Arthur J. O.

1988 "Sahagun's informants on the nature of Tlalocan » in J. Klor de Alva, H. B. Nicholson and E. Quiñones Keber, eds, The Work of Bernardino de Sahagin. Pioneer Ethnographer of the Sixteenth-Century Aztec Mexico, Institute for Mesoamerican Studies, University at Albany, State University of New York, Albany N. Y., pp. 151-160, coll. "Studies on culture and society $\gg 4$.

BAUdot Georges et Tzvetan Todorov

1983 Récits aztèques de la Conquête, Seuil, Paris.

BIERHORST John

1985

Cantares Mexicanos. Songs of the Aztecs, Stanford University Press, Stanford.

Codex Aubin

1979 [1902] Códice Aubin. Manuscrito azteca de la biblioteca real de Berlin, Anales en mexicano y geroglificos desde la salida de la tribu de Aztlan hasta la muerte de Cuauhtemoc, Bernardino de Jesús Quíroz, ed., Editorial Innovación, México.

Codex Vaticanus $A$

1964-1967 in Antigüedades de Mexico basadas en la recopilación de Lord Kingsborough, vol. 3, pp. 7-314, México.

Codex de Florence

1950-1981 Florentine Codex: General History of the Things of New Spain, Fray Bernardino de Sahagun, translated from the Aztec into English, with notes and illustrations by Charles E. Dibble and Arthur J. O. Anderson, 2 vols, The School of American Research and the University of Utah, Santa Fe, New Mexico.

Garibay Angel María K.

1964-1966 Poesía nahuatl, 3 vols, UNAM, México.

Graulich Michel

1987 Mythes et rituels du Mexique ancien pré-hispanique, Académie Royale de Belgique, Mémoire de la Classe de Lettres, Bruxelles. 
1991

"Les signes avant-coureurs de la chute de l'empire aztèque » in Alain Dierkens, ed., Apparitions et miracles, Éditions de l'université de Bruxelles, pp. 139-151.

León-Portilla Miguel y Librado Silva Galeana

1991 Huehuehtlahtolli, testimonios de la antigua palabra. SEP, FCE, México.

López Austin Alfredo

1984 Cuerpo humano e ideología. Las concepciones de los antiguos nahuas, 2 vols, IIA, UNAM, México.

Mendieta Fray Jerónimo de

$1980 \quad$ Historia eclesiástica indiana, Porrúa, México.

Muñoz Camargo Diego

1892 Historia de Tlaxcala, Alfredo Chavero, ed., México.

Primeros Memoriales

1997 Paleography of Nahuatl Text and English Translation, by Thelma Sullivan. University of Oklahoma Press and Real Academia de la Historia, Norman and Madrid.

1907

Ed. Facs. de Francisco del Paso y Troncoso, vol. vI, Fototipia de Hauset y Menet, Madrid.

RAGOT Nathalie

1999 Les au-delàs aztèques. Approches des conceptions sur la mort et le devenir des morts (Mexique), thèse de doctorat présentée à l'École pratique des hautes études, Paris.

Relation de Michoacan (La)

1984 J.-M. G. Le Clézio, éd., Gallimard, Paris.

SAHAGún Fray Bernardino de

1992 Historia general de las cosas de la Nueva España, Angel María Garibay, ed., Porrúa, México.

ToDORov Tzvetan

1982 La Conquête de l'Amérique. La question de l'autre, Seuil, Paris.

TORQuemada Fray Juan de

1975-1983 Monarquía Indiana : de los veinte y un libros rituales y monarquía indiana con el origen y guerras de los indios occidentales, de sus poblazones, descubrimiento, conquista, conversion y otras cosas maravillosas de la misma tierra, Miguel León-Portilla, ed., UNAM, IIH, México.

1986 Monarquía Indiana, Miguel León-Portilla, ed., Porrúa, México, 3 vols, coll. « Biblioteca Porrúa » 41. 